\title{
Interference Adsorption of Cadmium with a Variety of Pollutants in Sediments Based on Fractional Factorial Design (Resolution V)
}

\author{
Wenwen Gu${ }^{1,2}$, Bingchuan Cheng ${ }^{1,2}$, Yu Li ${ }^{1,2 *}$ \\ ${ }^{1}$ Resource and Environment Academy, North China Electric Power University, \\ Beijing 102206, China \\ ${ }^{2}$ The State Key Laboratory of Regional Optimization of Energy Systems, North China Electric Power University, \\ Beijing 102206, China
}

Received: 25 November 2015

Accepted: 16 August 2016

\begin{abstract}
The characteristics of composite cadmium contamination via adsorption onto the surficial sediments in a pesticide (dimethoate, metalaxyl, atrazine, malathion, and prometryn)/heavy metal (copper, zinc, lead, cadmium, and nickel) composite contamination system were examined. To do this, a confounding designassisted resolution $\mathrm{V}$ of $2^{10-3}$ fractional factorial design method composed of a fixed effects model, a multiple linear regression model, and the best subset regression modeling methods was used to identify the main effects and second-order interaction effects of the aforementioned pollutants. Overall, $87.08 \%$ of the total contribution to cadmium adsorption derived from the main effect, and the main effects of copper, lead, zinc, and dimethoate had a significant antagonistic effect on cadmium adsorption on the sediments in the order of: copper $(17.41 \%)>$ lead $(13.09 \%)>$ zinc $(10.06 \%)>$ dimethoate $(5.03 \%)$, while the main effects of cadmium $(41.49 \%)$ had a significant synergistic effect. Moreover, $12.92 \%$ of the total contribution to cadmium adsorption was attributed to second-order interaction effects (zinc*nickel and copper*zinc), with zinc*nickel (4.57\%) having a significant antagonistic effect and copper*zinc (8.35\%) having a significant synergistic effect on cadmium adsorption on the sediments. When compared with resolution IV of the $2^{10-5}$ fractional factorial design method, the freedom of resolution $V$ of the $2^{10-3}$ fractional factorial design method increased from 21 to 45 . This showed that resolution $V$ of the $2^{10-3}$ fractional factorial design method can significantly distinguish the aliases of the second-order interaction effects related to the objective pollutant cadmium. Also, the total contribution to cadmium adsorption of the second-order interaction effects decreased from $61.48 \%$ to $12.92 \%$, indicating that resolution IV of the $2^{10-5}$ fractional factorial design method overestimates the second-order interaction effect on cadmium adsorption on sediments.
\end{abstract}

Keywords: cadmium, heavy metals, pesticides, competitive adsorption, fractional factorial design

*e-mail: liyuxx8@hotmail.com 


\section{Introduction}

With the rapid development of agriculture and industry, pollution problems have become increasingly prominent [1]. Pesticides and heavy metals discharged into the environment accumulate [2] and later cause serious pollution to the atmosphere [3], water [4], and soil [5], while threatening the health of animals, plants, and humans [6-7]. There are few instances of only one pollutant or pollution source in the environment [8], and interactions between two types of pollutants often lead to further contamination by changing the environmental behavior and eco-toxicity, and forming a combined pollution [9].

Sediment is a type of small particle that can be moved by fluid flow and eventually become a layer of solid particles at the bottom of an aquatic system. Sediment is an important carrier leading to the migration of many pollutants in natural surfaces, as well as a major destination of pollutants in water [10]. Sediment can accumulate a variety of heavy metals, pesticides, and other pollutants from aquatic environments [11]. Scholars from various countries have conducted many studies regarding the adsorption behavior of heavy metals and pesticides in the water-sediment interface. These include investigations of pesticides and heavy metal contents of different geographical sediments [12-14], the mechanism of sediment adsorption on pollutants, and the mechanism of sediment adsorption on pollutants under combined pollution cases [15]. Guo et al. measured the effects of $\mathrm{Cr}$ (VI) and As (V) on lindane sorption and found that more lindane is adsorbed by biofilms than suspended particles and sediments on a total mass basis, whereas the sediments had a higher lindane sorption capacity per unit mass of organic carbon than suspended particles and biofilms. Cooccurring Cr (VI) or As (V) decreased the lindane sorption on the biofilms by about $48 \%$ [16].

Currently, the main difficulty in such studies is that there are many different types of pollutants in nature, making the combined fouling mechanism extremely complex. Cheng et al. used a resolution IV fractional factorial design method to explore the combined pollution rules of malathion and a variety of pollutants in sediment [17]. However, they could not solve the mixed phenomenon between the main effects and the second-order interaction.

Cadmium is a toxic heavy metal that is extremely harmful to humans and other mammalian species [18] and that is present in air, soil, sediments, water, and smoke. Following intake, cadmium accumulates in multiple organs and tissues - particularly the liver and kidneys [19]. In this study, we used a resolution V $2^{10-3}$ fractional factorial design method to reveal the composite contamination characteristics of cadmium adsorption onto the surficial sediments in a pesticide (dimethoate, metalaxyl, atrazine, malathion, and prometryn)/heavy metal (copper, zinc, lead, cadmium, and nickel) composite contamination system. This study provides theoretical support for revealing the combined pollution mechanism between pesticides and heavy metals.

\section{Materials and Methods}

\author{
Sediment Sample Collection \\ and Pretreatment
}

Sediment samples were collected from the Songhua River in Jilin, China. Samples were collected from the surface layer to a depth of $5 \mathrm{~cm}$ using a digging type sampler. One sample was collected every $20 \mathrm{~cm}$ from around the sampling point, and four samples were collected and then composited into a single sample.

\section{Experimental Methods and Designs}

Five pesticides - dimethoate (A), metalaxyl (B), atrazine (C), malathion (D), and prometryn (E) - and five heavy metals - copper $(F)$, cadmium $(G)$, lead $(H)$, zinc $(\mathrm{J})$, and nickel $(\mathrm{K})$ - were selected as experimental factors. The heavy metal $\mathrm{Cd}$ was the target pollutant, and the main effects and second-order interaction effects of pollutant adsorption on the sediments to the targets were investigated. The factor levels are shown in Table 1. In this experiment, a $2^{10-3}$ resolution $\mathrm{V}$ fractional factorial experiment with a minimum low-order mixture was used and 128 was treated as a unit and set parallel to the sample, resulting in a total of 384 group processing. Table 2 shows a $2^{10-3}$ fractional factorial experimental table established using statistical analysis software. Based on the principles of fractional factorial design in Minitab, the interactions of dimethoate (A), metalaxyl (B), atrazine (C), malathion (D), prometryn $(\mathrm{E}), \mathrm{Cd}(\mathrm{G})$, and $\mathrm{Cu}(\mathrm{F})$, and the form of the generating element, the concentrations of $\mathrm{Pb}(\mathrm{H}), \mathrm{Zn}$ $(\mathrm{J})$, and $\mathrm{Ni}(\mathrm{K})$ are determined as follows: $\mathrm{H}=\mathrm{ABCG}$, $\mathrm{J}=\mathrm{BCDE}$, and $\mathrm{K}=\mathrm{ACDF}$.

A mixed solution of 10 pollutants at different concentrations was prepared in accordance with Table 2. Next, $20 \mathrm{ml}$ of each of the 384 mixed solutions was placed into a conical flask containing $0.1000 \pm 0.0001 \mathrm{~g}$ of sediment samples. Each solution was then oscillated at room temperature for $48 \mathrm{~h}$, after which the solution was passed through filter paper with a pore size of $0.22 \mu \mathrm{m}$. Subsequently, the concentrations of pesticides and heavy metals in each solution were measured using HPLC and a flame atomic absorption spectrophotometer. In addition, the initial concentrations of the pollutants were measured. Finally, the amounts of pollutants adsorbed were determined by differential subtraction calculations.

According to the principle of the resolution $\mathrm{V}$ fractional factorial design method, the generators of the main effects and the second-order interaction effects were determined, and the aliases of the main effects and the second-order interaction effects were found to be as follows:

- Aliases of the main effects: $\mathrm{A}=\mathrm{BCGH}=\mathrm{CDFK}$, $\mathrm{B}=\mathrm{ACGH}=\mathrm{CDEJ}, \mathrm{C}=\mathrm{ABGH}=\mathrm{ADFK}=\mathrm{BDEJ}$, $\mathrm{D}=\mathrm{ACFK}=\mathrm{BCEJ}, \mathrm{E}=\mathrm{BCDJ}, \mathrm{F}=\mathrm{ACDK}$, $\mathrm{G}=\mathrm{ABCH}, \mathrm{H}=\mathrm{ABCG}, \mathrm{J}=\mathrm{BCDE}, \mathrm{K}=\mathrm{ACDF}$. 
Table 1. High and low levels of five heavy metals and five pesticides ( $\mu \mathrm{mol} / \mathrm{L})$.

\begin{tabular}{|c|c|c|c|c|c|}
\hline Level & Dimethoate & Metalaxyl & Atrazine & Malathion & Prometryn \\
\hline Low (-) & 10 & 10 & 10 & 10 & 10 \\
\hline High (+) & 25 & 25 & 25 & 25 & 25 \\
\hline Level & $\mathrm{Cu}$ & $\mathrm{Cd}$ & $\mathrm{Pb}$ & $\mathrm{Zn}$ & $\mathrm{Ni}$ \\
\hline Low (-) & 60 & 60 & 150 & 150 & 60 \\
\hline High (+) & 150 & 150 & & & 150 \\
\hline
\end{tabular}

Table 2. $2^{10-3}$ fractional factorial design of 10 pollutants adsorbed onto the sediments.

\begin{tabular}{|c|c|c|c|c|c|c|c|c|c|c|}
\hline & Dimethoate & Metalaxyl & Atrazine & Malathion & Prometryn & $\mathrm{Cu}$ & $\mathrm{Cd}$ & $\mathrm{Pb}$ & $\mathrm{Zn}$ & $\mathrm{Ni}$ \\
\hline 1 & - & + & + & - & - & - & + & - & + & - \\
\hline 2 & + & + & + & - & + & - & - & - & - & + \\
\hline 3 & - & + & - & + & - & - & + & + & + & - \\
\hline 4 & + & - & + & - & - & + & + & - & - & - \\
\hline 5 & + & - & - & - & + & - & + & + & - & - \\
\hline 6 & - & - & + & + & + & - & + & + & - & + \\
\hline 7 & + & + & - & + & + & + & - & + & - & - \\
\hline 8 & - & + & - & + & - & + & - & - & + & + \\
\hline 9 & - & - & - & - & + & - & - & + & - & + \\
\hline 10 & + & + & - & - & - & + & + & - & - & + \\
\hline 11 & - & + & - & + & + & + & - & - & - & + \\
\hline 12 & - & + & + & + & + & + & + & - & + & - \\
\hline 13 & + & - & + & + & + & - & - & + & - & - \\
\hline 14 & + & + & - & - & + & - & + & - & + & - \\
\hline 15 & - & - & + & - & - & - & + & + & - & - \\
\hline 16 & - & + & + & + & - & - & - & + & - & + \\
\hline 17 & + & - & - & - & - & - & + & + & + & - \\
\hline 18 & - & + & + & - & - & + & + & - & + & + \\
\hline 19 & + & + & + & + & - & + & - & - & - & + \\
\hline 20 & - & + & - & - & + & - & - & - & + & + \\
\hline 21 & - & - & + & - & - & + & + & + & - & + \\
\hline 22 & - & - & - & + & + & - & - & + & + & - \\
\hline 23 & + & + & - & + & - & - & - & + & + & + \\
\hline 24 & - & + & - & + & + & - & - & - & - & - \\
\hline 25 & - & + & + & + & - & - & + & - & - & + \\
\hline 26 & + & + & - & - & - & + & - & + & - & + \\
\hline 27 & + & + & + & + & + & - & - & - & + & - \\
\hline 28 & + & - & - & + & + & + & - & - & + & - \\
\hline 29 & - & - & + & - & - & - & - & - & - & - \\
\hline 30 & - & - & - & - & + & + & + & - & - & - \\
\hline 31 & + & + & + & + & + & - & + & + & + & - \\
\hline
\end{tabular}


Table 2. Continued.

\begin{tabular}{|c|c|c|c|c|c|c|c|c|c|c|}
\hline 32 & - & - & - & - & - & - & - & + & + & + \\
\hline 33 & + & + & + & - & - & + & - & - & + & - \\
\hline 34 & - & + & - & - & + & + & - & - & + & - \\
\hline 35 & + & + & - & - & - & - & + & - & - & - \\
\hline 36 & - & + & + & - & - & + & - & + & + & + \\
\hline 37 & - & - & - & + & - & - & - & + & - & - \\
\hline 38 & + & - & - & - & - & + & + & + & + & + \\
\hline 39 & + & + & - & - & - & - & - & + & - & - \\
\hline 40 & - & - & - & + & - & + & + & - & - & + \\
\hline 41 & + & + & - & - & + & + & + & - & + & + \\
\hline 42 & - & - & + & + & - & - & + & + & + & + \\
\hline 43 & - & - & + & - & + & + & + & + & + & + \\
\hline 44 & + & - & - & - & - & + & - & - & + & + \\
\hline 45 & + & + & + & - & + & + & + & + & - & - \\
\hline 46 & - & + & - & + & + & - & + & + & - & - \\
\hline 47 & - & - & - & - & - & + & - & + & + & - \\
\hline 48 & + & + & + & + & - & - & - & - & - & - \\
\hline 49 & - & + & - & + & - & - & - & - & + & - \\
\hline 50 & - & - & + & + & - & - & - & - & + & + \\
\hline 51 & + & + & - & + & - & + & - & + & + & - \\
\hline 52 & + & + & - & - & + & + & - & + & + & + \\
\hline 53 & + & - & - & + & - & + & - & - & - & - \\
\hline 54 & - & - & + & + & + & - & - & - & - & + \\
\hline 55 & - & - & - & + & - & + & - & + & - & + \\
\hline 56 & + & + & + & - & + & - & + & + & - & + \\
\hline 57 & - & + & + & + & + & - & + & - & + & + \\
\hline 58 & + & - & + & - & - & - & - & + & - & + \\
\hline 59 & + & + & - & + & + & - & - & + & - & + \\
\hline 60 & + & + & + & + & + & + & + & + & + & + \\
\hline 61 & - & + & - & - & - & + & + & + & - & - \\
\hline 62 & + & - & + & + & + & + & - & + & - & + \\
\hline 63 & + & - & - & - & + & + & + & + & - & + \\
\hline 64 & + & - & + & - & + & + & + & - & + & - \\
\hline 65 & - & - & - & + & - & - & + & - & - & - \\
\hline 66 & + & + & - & + & + & - & + & - & - & + \\
\hline 67 & + & - & + & - & + & + & - & + & + & - \\
\hline 68 & - & + & - & - & - & + & - & - & - & - \\
\hline 69 & + & + & + & - & - & + & + & + & + & - \\
\hline 70 & - & - & + & + & - & + & - & - & + & - \\
\hline 71 & - & - & - & - & - & + & + & - & + & - \\
\hline 72 & - & + & + & - & + & + & + & - & - & + \\
\hline
\end{tabular}


Table 2. Continued.

\begin{tabular}{|c|c|c|c|c|c|c|c|c|c|c|}
\hline 73 & + & + & + & + & - & + & + & + & - & + \\
\hline 74 & - & + & + & - & - & - & - & + & + & - \\
\hline 75 & - & - & - & - & + & + & - & + & - & - \\
\hline 76 & - & + & - & - & + & - & + & + & + & + \\
\hline 77 & - & - & + & - & + & - & - & - & + & - \\
\hline 78 & + & - & + & + & - & - & - & + & + & - \\
\hline 79 & + & - & - & + & + & - & - & - & + & + \\
\hline 80 & + & - & + & - & - & - & + & - & - & + \\
\hline 81 & + & + & - & - & + & - & - & + & + & - \\
\hline 82 & - & - & + & - & + & - & + & + & + & - \\
\hline 83 & + & - & + & + & + & + & + & - & - & + \\
\hline 84 & + & - & - & + & - & + & + & + & - & - \\
\hline 85 & + & - & - & + & + & - & + & + & + & + \\
\hline 86 & + & - & + & + & - & + & - & + & + & + \\
\hline 87 & - & - & - & + & + & + & + & - & + & + \\
\hline 88 & - & + & + & - & + & - & + & - & - & - \\
\hline 89 & - & - & - & - & + & - & + & - & - & + \\
\hline 90 & + & - & - & - & + & - & - & - & - & - \\
\hline 91 & + & - & + & + & + & - & + & - & - & - \\
\hline 92 & + & + & + & + & + & + & - & - & + & + \\
\hline 93 & + & - & + & + & - & - & + & - & + & - \\
\hline 94 & + & + & - & + & - & - & + & - & + & + \\
\hline 95 & + & + & - & + & - & + & + & - & + & - \\
\hline 96 & + & - & + & - & + & - & - & + & + & + \\
\hline 97 & + & - & - & + & + & + & + & + & + & - \\
\hline 98 & + & - & + & - & - & + & - & + & - & - \\
\hline 99 & + & - & - & - & - & - & - & - & + & - \\
\hline 100 & - & + & - & - & + & + & + & + & + & - \\
\hline 101 & - & + & + & + & + & - & - & + & + & + \\
\hline 102 & - & + & + & + & + & + & - & + & + & - \\
\hline 103 & - & + & - & - & - & - & + & + & - & + \\
\hline 104 & - & + & + & - & + & + & - & + & - & + \\
\hline 105 & - & + & - & + & - & + & + & + & + & + \\
\hline 106 & - & - & + & + & - & + & + & + & + & - \\
\hline 107 & + & - & - & + & - & - & - & - & - & + \\
\hline 108 & - & + & - & - & - & - & - & - & - & + \\
\hline 109 & - & - & + & + & + & + & + & + & - & - \\
\hline 110 & - & + & + & + & - & + & + & - & - & - \\
\hline 111 & - & - & - & + & + & - & + & - & + & - \\
\hline 112 & - & + & + & + & - & + & - & + & - & - \\
\hline 113 & + & - & - & + & - & - & + & + & - & + \\
\hline
\end{tabular}


Table 2. Continued.

\begin{tabular}{|l|l|l|l|l|l|l|l|l|l|l|}
\hline 114 & - & - & + & - & + & + & - & - & + & + \\
\hline 115 & + & + & + & + & - & - & + & + & - & - \\
\hline 116 & + & + & + & - & - & - & + & + & + & + \\
\hline 117 & + & - & + & + & - & + & + & - & + & + \\
\hline 118 & - & - & + & - & - & + & - & - & - & + \\
\hline 119 & - & + & + & - & + & - & - & + & - & - \\
\hline 120 & - & + & - & + & + & + & + & + & - & + \\
\hline 121 & - & - & - & + & + & + & - & + & + & + \\
\hline 122 & + & + & + & - & - & - & - & - & + & + \\
\hline 123 & + & + & + & - & + & + & - & - & - & - \\
\hline 124 & + & - & + & - & + & - & + & - & + & + \\
\hline 125 & - & - & - & - & - & - & + & - & + & + \\
\hline 126 & + & + & - & + & + & + & + & - & - & - \\
\hline 127 & - & - & + & + & + & + & - & - & - & - \\
\hline 128 & + & - & - & - & + & + & - & - & - \\
\hline
\end{tabular}

- Aliases of the second-order interaction effects: $\mathrm{AB}=\mathrm{CGH}=\mathrm{EFJK}, \mathrm{AC}=\mathrm{BGH}=\mathrm{DFK}, \mathrm{AD}=\mathrm{CFK}$ $=\mathrm{EGHJ}, \mathrm{AE}=\mathrm{BFJK}=\mathrm{DGHJ}, \mathrm{AF}=\mathrm{CDK}=\mathrm{BEJK}$, $\mathrm{AG}=\mathrm{BCH}=\mathrm{DEHJ}, \mathrm{AH}=\mathrm{BCG}=\mathrm{DEGJ}, \mathrm{AJ}=\mathrm{BEFK}$ $=\mathrm{DEGH}, \mathrm{AK}=\mathrm{CDF}=\mathrm{BEFJ}, \mathrm{BC}=\mathrm{AGH}=\mathrm{DEJ}$, $\mathrm{BD}=\mathrm{CEJ}=\mathrm{FGHK}, \mathrm{BE}=\mathrm{CDJ}=\mathrm{AFJK}, \mathrm{BF}=\mathrm{AEJK}$ $=\mathrm{DGHK}, \mathrm{BG}=\mathrm{ACH}=\mathrm{DFHK}, \mathrm{AK}=\mathrm{CDF}=\mathrm{BEFJ}$, $\mathrm{BC}=\mathrm{AGH}=\mathrm{DEJ}, \mathrm{BD}=\mathrm{CEJ}=\mathrm{FGHK}, \mathrm{BE}=\mathrm{CDJ}=$ $\mathrm{AFJK}, \mathrm{BF}=\mathrm{AEJK}=\mathrm{DGHK}, \mathrm{BG}=\mathrm{ACH}=\mathrm{DFHK}$, $\mathrm{BH}=\mathrm{ACG}=\mathrm{DFGK}, \mathrm{BJ}=\mathrm{CDE}=\mathrm{AEFK}, \mathrm{BK}=\mathrm{AEFJ}$ $=\mathrm{DFGH}, \mathrm{CD}=\mathrm{AFK}=\mathrm{BEJ}, \mathrm{CE}=\mathrm{BDJ}, \mathrm{CF}=\mathrm{ADK}$, $\mathrm{CG}=\mathrm{ABH}, \mathrm{CH}=\mathrm{ABG}, \mathrm{CJ}=\mathrm{BDE}, \mathrm{CK}=\mathrm{ADF}, \mathrm{DE}$ $=\mathrm{BCJ}=\mathrm{AGHJ}, \mathrm{DF}=\mathrm{ACK}=\mathrm{BGHK}, \mathrm{DG}=\mathrm{AEHJ}=$ $\mathrm{BFHK}, \mathrm{DH}=\mathrm{AEGJ}=\mathrm{BFGK}, \mathrm{DJ}=\mathrm{BCE}=\mathrm{AEGH}$, $\mathrm{DK}=\mathrm{ACF}=\mathrm{BFGH}, \mathrm{EF}=\mathrm{ABJK}, \mathrm{EG}=\mathrm{ADHJ}, \mathrm{EH}=$ $\mathrm{ADGJ}, \mathrm{EJ}=\mathrm{BCD}=\mathrm{ABFK}=\mathrm{ADGH}, \mathrm{EK}=\mathrm{ABFJ}, \mathrm{FG}$ $=\mathrm{BDHK}, \mathrm{FH}=\mathrm{BDGK}, \mathrm{EG}=\mathrm{ADHJ}, \mathrm{EH}=\mathrm{ADGJ}, \mathrm{EJ}$ $=\mathrm{BCD}=\mathrm{ABFK}=\mathrm{ADGH}, \mathrm{EK}=\mathrm{ABFJ}, \mathrm{FG}=\mathrm{BDHK}$, $\mathrm{FH}=\mathrm{BDGK}, \mathrm{FJ}=\mathrm{ABEK}, \mathrm{FK}=\mathrm{ACD}=\mathrm{ABEJ}=$ $\mathrm{BDGH}, \mathrm{GH}=\mathrm{ABC}=\mathrm{ADEJ}=\mathrm{BDFK}, \mathrm{GJ}=\mathrm{ADEH}$, $\mathrm{GK}=\mathrm{BDFH}, \mathrm{HJ}=\mathrm{ADEG}, \mathrm{HK}=\mathrm{BDFG}, \mathrm{JK}=\mathrm{ABEF}$.

As shown above, the alias structure of interactions could be used to distinguish the main effects from the second-order interaction effects completely by fractional factorial design of resolution $\mathrm{V}$. In addition, the response value of each main effect and the second-order interaction effect were estimated.

\section{Data Processing and Analysis}

Experimental design and statistical analysis were performed using the experimental design module in the Minitab software package (Design of Experiment). Before using a fixed effects model, a fit test (i.e., normal assumption, independence assumption of residuals, and homogeneity assumption of the variance) was carried out [20]. The model was only considered suitable if it met the above conditions. Next, we set the adsorption capacity of $\mathrm{Cd}$ onto the sediment as the dependent variable and the 10 types of main pollutant concentration effects and the second-order interaction effects as the independent variables. This was done to establish the multiple linear regression adsorption model and the best subset regression model.

Table 3. Analysis of variance of Cd adsorption effects.

\begin{tabular}{|c|c|c|c|c|c|}
\hline Source & DF & SS & MS & F & P \\
\hline Main effect & 10 & $1,487.59$ & 148.76 & 45.84 & 0.000 \\
\hline Second-order effect & 45 & 211.37 & 4.70 & 1.45 & 0.080 \\
\hline Residual error & 72 & 233.67 & 3.25 & & \\
\hline Total & 127 & $1,932.63$ & & & \\
\hline
\end{tabular}


Table 4. Estimates of main and second-order effects of pollutant concentrations affecting $\mathrm{Cd}$ in the sorption of heavy metals and the pesticide system.

\begin{tabular}{|c|c|c|c|c|}
\hline Factor & Estimate of effect & Coefficient & $\mathrm{T}$ & $\mathrm{P}$ \\
\hline Constant & 9.568 & 0.1592 & 0.000 & \\
\hline Dimethoate & -0.706 & -0.353 & -2.22 & 0.030 \\
\hline Metalaxyl & -0.155 & -0.078 & -0.49 & 0.627 \\
\hline Atrazine & 0.398 & 0.199 & 1.25 & 0.215 \\
\hline Malathion & 0.432 & 0.216 & 1.36 & 0.179 \\
\hline Prometryn & -0.190 & -0.095 & -0.60 & 0.552 \\
\hline $\mathrm{Cu}$ & -2.442 & -1.221 & -7.67 & 0.000 \\
\hline $\mathrm{Cd}$ & 5.821 & 2.910 & 18.28 & 0.000 \\
\hline $\mathrm{Pb}$ & -1.836 & -0.918 & -5.76 & 0.000 \\
\hline $\mathrm{Zn}$ & -1.412 & -0.706 & -4.43 & 0.000 \\
\hline $\mathrm{Ni}$ & -0.612 & -0.306 & -1.92 & 0.059 \\
\hline Dimethoate*Metalaxyl & -0.483 & -0.242 & -1.52 & 0.134 \\
\hline Dimethoate*Atrazine & 0.283 & 0.141 & 0.89 & 0.378 \\
\hline Dimethoate*Malathion & 0.144 & 0.072 & 0.45 & 0.653 \\
\hline Dimethoate*Prometryn & 0.089 & 0.044 & 0.28 & 0.781 \\
\hline Dimethoate $* \mathrm{Cu}$ & 0.127 & 0.063 & 0.40 & 0.692 \\
\hline Dimethoate*Cd & -0.340 & -0.170 & -1.07 & 0.290 \\
\hline Dimethoate* $\mathrm{Pb}$ & 0.207 & 0.104 & 0.65 & 0.518 \\
\hline Dimethoate*Zn & -0.087 & -0.043 & -0.27 & 0.786 \\
\hline Dimethoate*Ni & 0.422 & 0.211 & 1.33 & 0.189 \\
\hline Metalaxyl*Atrazine & -0.137 & -0.068 & -0.43 & 0.669 \\
\hline Metalaxyl*Malathion & -0.477 & -0.238 & -1.50 & 0.139 \\
\hline Metalaxyl*Prometryn & 0.109 & 0.055 & 0.34 & 0.733 \\
\hline Metalaxyl* ${ }^{*} \mathrm{Cu}$ & 0.241 & 0.121 & 0.76 & 0.452 \\
\hline Metalaxyl*Cd & -0.219 & -0.110 & -0.69 & 0.493 \\
\hline Metalaxyl ${ }^{*} \mathrm{~Pb}$ & 0.170 & 0.085 & 0.53 & 0.595 \\
\hline Metalaxyl*Zn & -0.414 & -0.207 & -1.30 & 0.198 \\
\hline Metalaxyl*Ni & -0.173 & -0.087 & -0.54 & 0.588 \\
\hline Atrazine*Malathion & -0.299 & -0.149 & -0.94 & 0.351 \\
\hline Atrazine*Prometryn & 0.415 & 0.207 & 1.30 & 0.197 \\
\hline Atrazine $* \mathrm{Cu}$ & -0.093 & -0.047 & -0.29 & 0.770 \\
\hline Atrazine $* \mathrm{Cd}$ & 0.510 & 0.255 & 1.60 & 0.114 \\
\hline Atrazine $* \mathrm{~Pb}$ & -0.128 & -0.064 & -0.40 & 0.690 \\
\hline Atrazine*Zn & -0.546 & -0.273 & -1.71 & 0.091 \\
\hline Atrazine* $\mathrm{Ni}$ & -0.311 & -0.155 & -0.98 & 0.332 \\
\hline Malathion*Prometryn & -0.319 & -0.159 & -1.00 & 0.320 \\
\hline Malathion* $\mathrm{Cu}$ & 0.335 & 0.168 & 1.05 & 0.296 \\
\hline Malathion*Cd & 0.081 & 0.041 & 0.26 & 0.799 \\
\hline Malathion* $\mathrm{Pb}$ & 0.162 & 0.081 & 0.51 & 0.612 \\
\hline
\end{tabular}


Table 4. Continued.

\begin{tabular}{|c|c|c|c|c|}
\hline Malathion*Zn & -0.292 & -0.146 & -0.92 & 0.363 \\
\hline Malathion*Ni & -0.198 & -0.099 & -0.62 & 0.536 \\
\hline Prometryn*Cu & 0.335 & 0.167 & 1.05 & 0.297 \\
\hline Prometryn*Cd & 0.369 & 0.184 & 1.16 & 0.251 \\
\hline Prometryn*Pb & 0.133 & 0.066 & 0.42 & 0.679 \\
\hline Prometryn*Zn & 0.478 & 0.239 & 1.50 & 0.137 \\
\hline Prometryn*Ni & -0.367 & -0.183 & -1.15 & 0.253 \\
\hline $\mathrm{Cu} * \mathrm{Cd}$ & -0.205 & -0.103 & -0.64 & 0.521 \\
\hline $\mathrm{Cu} * \mathrm{~Pb}$ & -0.512 & -0.256 & -1.61 & 0.112 \\
\hline $\mathrm{Cu} * \mathrm{Zn}$ & 1.171 & 0.585 & -0.38 & 0.000 \\
\hline $\mathrm{Cu} * \mathrm{Ni}$ & -0.123 & -0.061 & -1.85 & 0.702 \\
\hline $\mathrm{Cd} * \mathrm{~Pb}$ & -0.588 & -0.294 & -1.53 & 0.069 \\
\hline $\mathrm{Cd} * \mathrm{Zn}$ & -0.487 & -0.244 & -1.69 & 0.130 \\
\hline $\mathrm{Cd} * \mathrm{Ni}$ & -0.538 & -0.269 & 1.96 & 0.096 \\
\hline $\mathrm{Pb} * \mathrm{Zn}$ & 0.625 & 0.313 & 0.19 & 0.053 \\
\hline $\mathrm{Pb} * \mathrm{Ni}$ & 0.062 & -0.031 & -2.01 & 0.846 \\
\hline $\mathrm{Zn} * \mathrm{Ni}$ & -0.641 & & & 0.048 \\
\hline
\end{tabular}

\section{Analysis of Variance}

Effect estimates and analysis of variance were employed to analyze the effects of the pollutant factor concentration in the heavy metal/pesticide coexistence system on the adsorption capacity of the sediment to $\mathrm{Cd}$. Next, the significant impact factors were filtered out using $\mathrm{P}<0.05$ to indicate significance. The analysis of variance results are shown in Table 3, and the effect estimates are presented in Table 4.

As shown in Table 3, analysis of variance showed that the main effect was significant, while the secondorder interaction effect was not, indicating that the main effects influenced the absorption of cadmium on sediment. When compared with resolution IV of the $2^{10-5}$ fractional factorial design method, the freedom increases from 21 to 45 , and the alias structures of second-order interaction effects are broken in the resolution $\mathrm{V}$ experiment, showing that resolution $\mathrm{V}$ of the $2^{10-3}$ fractional factorial design method can distinguish the alias structure of the second-order interaction effect significantly [17].

As shown in Table 4, the main effects of Cd, as well as the second-order interaction effects of cadmium* $\mathrm{Zn}$, had significant synergistic effects on cadmium adsorption in the sediment. The estimated effects were 5.821 and 1.171, respectively, while the main effects of factors that had significant antagonistic effects on $\mathrm{Cd}$ adsorption on the sediment were $\mathrm{Cu}=-2.442, \mathrm{~Pb}=-1.836, \mathrm{Zn}=-1.412$, dimethoate $=-0.706$, and $\mathrm{Ni}=-0.612$, and the secondorder interaction effect was $\mathrm{Zn} *$ nickel $=0.048$.

\section{Analysis of Contribution Rate}

The contribution rate of the significant main effects and second-order interaction effects to $\mathrm{Cd}$ adsorption reflected the composite contamination characteristics of $\mathrm{Cd}$ adsorption directly. Combined pollution effects are shown in Table 5.

Table 5 lists the calculated values of the main effects and the second-order interaction effects based on a fixed effects model, as well as the contribution rate of synergism and antagonism. As shown in the table, the contribution of the main effects and second-order interaction effects to the adsorption of Cd were $87.08 \%$ and $12.92 \%$, respectively, and the contribution rate of $\mathrm{Cd}$ was $41.49 \%$. Ma et al. pre-

Table 5. Combined pollution effects of pollutant fact concentration of $\mathrm{Cd}$ adsorption.

\begin{tabular}{|c|c|c|c|}
\hline \multirow{2}{*}{ Factor } & \multirow{2}{*}{$\begin{array}{c}\text { Estimate } \\
\text { of effect }\end{array}$} & \multicolumn{2}{|c|}{ Rate of contribution[\%] } \\
\cline { 3 - 4 } & 5.821 & 41.49 & \\
\hline $\mathrm{Cd}$ & synergism & antagonism \\
\hline $\mathrm{Cu}$ & -2.442 & & 17.41 \\
\hline $\mathrm{Pb}$ & -1.836 & & 13.09 \\
\hline $\mathrm{Zn}$ & -1.412 & & 10.06 \\
\hline Dimethoate & -0.706 & & 5.03 \\
\hline $\mathrm{Cu} \mathrm{Zn}$ & 1.171 & 8.35 & \\
\hline $\mathrm{Zn} * \mathrm{Ni}$ & -0.641 & & 4.57 \\
\hline Total & 14.029 & 49.84 & 50.16 \\
\hline
\end{tabular}


Table 6. Goodness of fit test for AT-MRAM (1).

\begin{tabular}{|c|c|c|c|}
\hline MRAM & $\mathrm{R}^{2}$ & Adjust $\mathrm{R}^{2}$ & Forecast $\mathrm{R}^{2}$ \\
\hline Value & $87.91 \%$ & $78.67 \%$ & $61.79 \%$ \\
\hline
\end{tabular}

Table 7. T-test for AT-MRAM (1).

\begin{tabular}{|c|c|c|c|}
\hline Factor & Coefficient & $\mathrm{T}$ & $\mathrm{P}$ \\
\hline Constant & 0.1592 & 0.000 & \\
\hline Dimethoate & -0.353 & -2.22 & 0.030 \\
\hline Metalaxyl & -0.078 & -0.49 & 0.627 \\
\hline Atrazine & 0.199 & 1.25 & 0.215 \\
\hline Malathion & 0.216 & 1.36 & 0.179 \\
\hline Prometryn & -0.095 & -0.60 & 0.552 \\
\hline $\mathrm{Cu}$ & -1.221 & -7.67 & 0.000 \\
\hline $\mathrm{Cd}$ & 2.910 & 18.28 & 0.000 \\
\hline $\mathrm{Pb}$ & -0.918 & -5.76 & 0.000 \\
\hline $\mathrm{Zn}$ & -0.706 & -4.43 & 0.000 \\
\hline $\mathrm{Ni}$ & -0.306 & -1.92 & 0.059 \\
\hline Dimethoate*Metalaxyl & -0.242 & -1.52 & 0.134 \\
\hline Dimethoate*Atrazine & 0.141 & 0.89 & 0.378 \\
\hline Dimethoate*Malathion & 0.072 & 0.45 & 0.653 \\
\hline Dimethoate*Prometryn & 0.044 & 0.28 & 0.781 \\
\hline Dimethoate $* \mathrm{Cu}$ & 0.063 & 0.40 & 0.692 \\
\hline Dimethoate*Cd & -0.170 & -1.07 & 0.290 \\
\hline Dimethoate $* \mathrm{~Pb}$ & 0.104 & 0.65 & 0.518 \\
\hline Dimethoate*Zn & -0.043 & -0.27 & 0.786 \\
\hline Dimethoate*Ni & 0.211 & 1.33 & 0.189 \\
\hline Metalaxyl*Atrazine & -0.068 & -0.43 & 0.669 \\
\hline Metalaxyl*Malathion & -0.238 & -1.50 & 0.139 \\
\hline Metalaxyl*Prometryn & 0.055 & 0.34 & 0.733 \\
\hline Metalaxyl*Cu & 0.121 & 0.76 & 0.452 \\
\hline Metalaxyl*Cd & -0.110 & -0.69 & 0.493 \\
\hline Metalaxyl*Pb & 0.085 & 0.53 & 0.595 \\
\hline Metalaxyl*Zn & -0.207 & -1.30 & 0.198 \\
\hline Metalaxyl*Ni & -0.087 & -0.54 & 0.588 \\
\hline Atrazine*Malathion & -0.149 & -0.94 & 0.351 \\
\hline Atrazine*Prometryn & 0.207 & 1.30 & 0.197 \\
\hline Atrazine $* \mathrm{Cu}$ & -0.047 & -0.29 & 0.770 \\
\hline Atrazine $* \mathrm{Cd}$ & 0.255 & 1.60 & 0.114 \\
\hline Atrazine $* \mathrm{~Pb}$ & -0.064 & -0.40 & 0.690 \\
\hline Atrazine* $\mathrm{Zn}$ & -0.273 & -1.71 & 0.091 \\
\hline Atrazine* $\mathrm{Ni}$ & -0.155 & -0.98 & 0.332 \\
\hline
\end{tabular}

Table 7. Continued.

\begin{tabular}{|c|c|c|c|}
\hline Malathion*Prometryn & -0.159 & -1.00 & 0.320 \\
\hline Malathion* $\mathrm{Cu}$ & 0.168 & 1.05 & 0.296 \\
\hline Malathion*Cd & 0.041 & 0.26 & 0.799 \\
\hline Malathion*Pb & 0.081 & 0.51 & 0.612 \\
\hline Malathion*Zn & -0.146 & -0.92 & 0.363 \\
\hline Malathion*Ni & -0.099 & -0.62 & 0.536 \\
\hline Prometryn $* \mathrm{Cu}$ & 0.167 & 1.05 & 0.297 \\
\hline Prometryn*Cd & 0.184 & 1.16 & 0.251 \\
\hline Prometryn $* \mathrm{~Pb}$ & 0.066 & 0.42 & 0.679 \\
\hline Prometryn*Zn & 0.239 & 1.50 & 0.137 \\
\hline Prometryn*Ni & -0.183 & -1.15 & 0.253 \\
\hline $\mathrm{Cu}^{*} \mathrm{Cd}$ & -0.103 & -0.64 & 0.521 \\
\hline $\mathrm{Cu} * \mathrm{~Pb}$ & -0.256 & -1.61 & 0.112 \\
\hline $\mathrm{Cu}^{*} \mathrm{Zn}$ & 0.585 & 3.68 & 0.000 \\
\hline $\mathrm{Cu} * \mathrm{Ni}$ & -0.061 & -0.38 & 0.702 \\
\hline $\mathrm{Cd} * \mathrm{~Pb}$ & -0.294 & -1.85 & 0.069 \\
\hline $\mathrm{Cd} * \mathrm{Zn}$ & -0.244 & -1.53 & 0.130 \\
\hline $\mathrm{Cd} * \mathrm{Ni}$ & -0.269 & -1.69 & 0.096 \\
\hline $\mathrm{Pb} * \mathrm{Zn}$ & 0.313 & 1.96 & 0.053 \\
\hline $\mathrm{Pb} * \mathrm{Ni}$ & 0.031 & 0.19 & 0.846 \\
\hline $\mathrm{Zn} * \mathrm{Ni}$ & -0.321 & -2.01 & 0.048 \\
\hline
\end{tabular}

viously revealed the combined pollution characteristics of $\mathrm{Cd}$ adsorption onto the surficial sediments by resolution IV of the $2^{10-5}$ fractional factorial design method. When compared with resolution IV, the total contri-bution to cadmium adsorption of the second-order interaction effect decreased from $61.48 \%$ to $12.92 \%$. This indicates that resolution $\mathrm{V}$ overestimates the second-order interaction effect of cadmium adsorption onto the sediments [21]. The single factor of $\mathrm{Cd}$ plays a critical role in inhibition of the absorption of $\mathrm{Cd}$. The sum of the contribution of synergism and antagonism was $49.84 \%$ and $50.16 \%$, respectively, revealing that the synergism and antagonism of $\mathrm{Cd}$ absorption reached a balanced state in the combined pollution system. The main effects of heavy metals had a greater influence than that of the pesticides. All of the heavy metals except $\mathrm{Cd}$ inhibit the absorption of $\mathrm{Cd}$.

\section{Establishing the Multiple Linear Regression Model}

The statistical module of the Minitab software was used to establish an adsorption model of multiple linear regression between the main effects of the adsorption capacity of $\mathrm{Cd}$ on the sediment with 10 types of pollutant factors and the second-order interaction effects. 
$G u W$., et al.

\begin{tabular}{|c|c|c|c|c|c|c|c|c|c|c|c|c|c|c|c|c|c|c|c|c|c|c|c|c|}
\hline $\begin{array}{l}\text { Dimethoate* } \\
\text { Ni }\end{array}$ & & & & & & & & & & & $x$ & $x$ & $x$ & $x$ & $x$ & $x$ & $x$ & $x$ & $x$ & $x$ & $x$ & $x$ & $x$ & $x$ \\
\hline $\begin{array}{c}\text { Dimethoate* } \\
\text { Zn }\end{array}$ & & & & & & & & & & & & & & & & & & & & & & & $x$ & $x$ \\
\hline $\begin{array}{c}\text { Dimethoate* } \\
\mathrm{Pb}\end{array}$ & & & & & & & & & & & & & & & $x$ & $x$ & $x$ & $x$ & $x$ & $x$ & $x$ & $x$ & $x$ & $x$ \\
\hline $\begin{array}{c}\text { Dimethoate* } \\
\text { Cd }\end{array}$ & & & & & & & & & & & & & $x$ & $x$ & $x$ & $x$ & $x$ & $x$ & $x$ & $x$ & $x$ & $x$ & $x$ & $x$ \\
\hline $\begin{array}{l}\text { Dimethoate* } \\
\mathrm{Cu}\end{array}$ & & & & & & & & & & & & & & & & & & & & $x$ & $x$ & $x$ & $x$ & $x$ \\
\hline $\begin{array}{l}\text { Dimethoate } \\
\text { *Prometryn }\end{array}$ & & & & & & & & & & & & & & & & & & & & & & $x$ & $x$ & $x$ \\
\hline $\begin{array}{l}\text { Dimethoate } \\
\text { *Malathion }\end{array}$ & & & & & & & & & & & & & & & & & & & $x$ & $x$ & $x$ & $x$ & $x$ & $x$ \\
\hline $\begin{array}{l}\text { Dimethoate } \\
* \text { Atrazine }\end{array}$ & & & & & & & & & & & & & & $x$ & $x$ & $x$ & $x$ & $x$ & $x$ & $x$ & $x$ & $x$ & $x$ & $x$ \\
\hline $\begin{array}{l}\text { Dimethoate } \\
\text { *Metalaxyl }\end{array}$ & & & & & & & & & $x$ & $x$ & $x$ & $x$ & $x$ & $x$ & $x$ & $x$ & $x$ & $x$ & $x$ & $x$ & $x$ & $x$ & $x$ & $x$ \\
\hline $\mathrm{Pb} * \mathrm{Ni}$ & & & & & & & & & & & & & & & & & & & & & & & & $x$ \\
\hline $\mathrm{Pb} * \mathrm{Zn}$ & & $x$ & $x$ & $x$ & $x$ & & $x$ & $x$ & $x$ & $x$ & $x$ & $x$ & $x$ & $x$ & $x$ & $x$ & $x$ & $x$ & $x$ & $x$ & $x$ & $x$ & $x$ & $x$ \\
\hline $\mathrm{Cd} * \mathrm{Ni}$ & & & & & $x$ & & $x$ & $x$ & $x$ & $x$ & $x$ & $x$ & $x$ & $x$ & $x$ & $x$ & $x$ & $x$ & $x$ & $x$ & $x$ & $x$ & $x$ & $x$ \\
\hline $\mathrm{Cd} * \mathrm{Zn}$ & & & & & & & & $x$ & $x$ & $x$ & $x$ & $x$ & $x$ & $x$ & $x$ & $x$ & $x$ & $x$ & $x$ & $x$ & $x$ & $x$ & $x$ & $x$ \\
\hline $\mathrm{Cd} * \mathrm{~Pb}$ & & & & $x$ & $x$ & & $x$ & $x$ & $x$ & $x$ & $x$ & $x$ & $x$ & $x$ & $x$ & $x$ & $x$ & $x$ & $x$ & $x$ & $x$ & $x$ & $x$ & $x$ \\
\hline $\mathrm{Cu} * \mathrm{Ni}$ & & & & & & & & & & & & & & & & & & & & & $x$ & $x$ & $x$ & $x$ \\
\hline $\mathrm{Cu} * \mathrm{~Pb}$ & & & & & & & $x$ & $x$ & $x$ & $x$ & $x$ & $x$ & $x$ & $x$ & $x$ & $x$ & $x$ & $x$ & $x$ & $x$ & $x$ & $x$ & $x$ & $x$ \\
\hline $\mathrm{Cu}^{*} \mathrm{Cd}$ & & & & & & & & & & & & & & & & $x$ & $x$ & $x$ & $x$ & $x$ & $x$ & $x$ & $x$ & $x$ \\
\hline $\mathrm{Ni}$ & & & $x$ & $x$ & $x$ & & $x$ & $x$ & $x$ & $x$ & $x$ & $x$ & $x$ & $x$ & $x$ & $x$ & $x$ & $x$ & $x$ & $x$ & $x$ & $x$ & $x$ & $x$ \\
\hline Prometryn & & & & & & & & & & & & & & & & & $x$ & $x$ & $x$ & $x$ & $x$ & $x$ & $x$ & $x$ \\
\hline Malathion & & & & & & & & & & $x$ & $x$ & $x$ & $x$ & $x$ & $x$ & $x$ & $x$ & $x$ & $x$ & $x$ & $x$ & $x$ & $x$ & $x$ \\
\hline Atrazine & & & & & & & & & & & & $x$ & $x$ & $x$ & $x$ & $x$ & $x$ & $x$ & $x$ & $x$ & $x$ & $x$ & $x$ & $x$ \\
\hline Metalaxyl & & & & & & & & & & & & & & & & & & $x$ & $x$ & $x$ & $x$ & $x$ & $x$ & $x$ \\
\hline Dimethoate & $x$ & $x$ & $x$ & $x$ & $x$ & & $x$ & $x$ & $x$ & $x$ & $x$ & $x$ & $x$ & $x$ & $x$ & $x$ & $x$ & $x$ & $x$ & $x$ & $x$ & $x$ & $x$ & $x$ \\
\hline S & 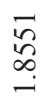 & $\begin{array}{l}n \\
\tilde{W} \\
\infty \\
\infty \\
-1\end{array}$ & 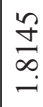 & $\begin{array}{l}\bar{\Xi} \\
\stackrel{\nabla}{-}\end{array}$ & $\begin{array}{l}0 \\
\infty \\
\alpha\end{array}$ & & $\begin{array}{c}\infty \\
\infty \\
0 \\
\\
\\
\end{array}$ & 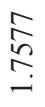 & 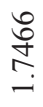 & 胥 & $\underset{\overbrace{}}{\approx}$ & 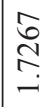 & $\stackrel{\infty}{\stackrel{\infty}{\sim}}$ & $\mid \begin{array}{l}8 \\
\mathbb{8} \\
\mathbb{2} \\
\end{array}$ & 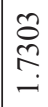 & 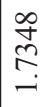 & 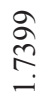 & $\mid \begin{array}{c}\overrightarrow{0} \\
\stackrel{+}{+} \\
\overrightarrow{-}\end{array}$ & 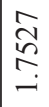 & $\frac{2}{2}$ & 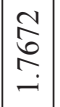 & $\stackrel{n}{\stackrel{n}{n}}$ & 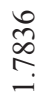 & $\underset{\curvearrowright}{\curvearrowright}$ \\
\hline Mallows Cp & $\underset{0}{0}$ & $\underset{\Xi}{ \pm}$ & 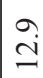 & $\stackrel{n}{=}$ & $\begin{array}{l}0 \\
0 \\
0\end{array}$ & & $\stackrel{0}{\circ}$ & \begin{tabular}{l}
0 \\
\hdashline \\
0
\end{tabular} & $\tilde{a}$ & $\dddot{n}$ & $\hat{a}$ & $\overrightarrow{0}$ & $\stackrel{\circ}{=}$ & 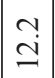 & $\ddot{\ddot{n}}$ & $\ddot{n}$ & $\ddot{\theta}$ & $\underset{\infty}{\infty}$ & $\dddot{\sim}$ & $\stackrel{n}{\mathrm{~N}}$ & $\stackrel{\sim}{\stackrel{\sim}{\sim}}$ & $\vec{i}$ & $\stackrel{0}{\infty}$ & O̊. \\
\hline $\begin{array}{l}\text { R-Sq } \\
\text { (ad) }\end{array}$ & $\stackrel{+}{\stackrel{r}{N}}$ & $\frac{9}{2}$ & $\underset{\sim}{\stackrel{\infty}{\sim}}$ & $\begin{array}{l}\infty \\
\infty \\
\infty\end{array}$ & 2 & & t. & $\hat{\Omega}$ & $\stackrel{\circ}{\infty}$ & சே. & $\begin{array}{l}n \\
\infty \\
\infty\end{array}$ & $\stackrel{+}{\dot{\infty}}$ & $\stackrel{+}{\dot{\infty}}$ & $\stackrel{+}{\dot{\infty}}$ & $\stackrel{n}{\infty}$ & $\underset{\infty}{\infty}$ & $\vec{\infty}$ & $\begin{array}{l}0 \\
\dot{\infty}\end{array}$ & $\stackrel{\infty}{2}$ & $\stackrel{0}{2}$ & $\ddot{n}$ & ח? & $\ddot{2}$ & $\underset{\infty}{\infty}$ \\
\hline $\mathrm{R}-\mathrm{Sq}$ & $\begin{array}{l}0 \\
\infty \\
\infty\end{array}$ & $\stackrel{n}{2}$ & হे & $\ddot{\infty}$ & ळे & & $\underset{\infty}{+}$ & $\stackrel{\infty}{\infty}$ & $\underset{\infty}{N}$ & ñ & $\begin{array}{l}\infty \\
\infty \\
\infty\end{array}$ & $\stackrel{\circ}{\infty}$ & $\stackrel{\sim}{\infty}$ & $\begin{array}{l}\dot{\infty} \\
\dot{\infty}\end{array}$ & $\stackrel{+}{\infty}$ & $\ddot{n}$ & $\begin{array}{l}0 \\
\infty\end{array}$ & $\begin{array}{l}0 \\
\dot{\infty} \\
\infty\end{array}$ & $\begin{array}{l}0 \\
\dot{\infty} \\
\infty\end{array}$ & $\dot{\infty}$ & $\stackrel{r}{\infty}$ & $\dot{\infty}$ & $\ddot{\infty}$ & ஸ் \\
\hline Vars & - & $N$ & $m$ & $\nabla$ & is & & 6 & $r$ & $\infty$ & $a$ & 으 & $=$ & $\simeq$ & 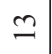 & \pm & 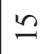 & $\stackrel{0}{-}$ & I & $\infty$ & 2 & ๙ & $\vec{\sim}$ & ป & 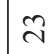 \\
\hline
\end{tabular}


Initially, all 10 pollutant factors and their second-order interaction terms were set as independent variables as follows:

$$
\begin{aligned}
& Y_{(\mathrm{Cd})}=9.57-\Gamma_{\text {Dimethoate }}(0.353) X_{\text {Dimethoate }}-\Gamma_{\text {Metalaxyl }}(0.078) \\
& X_{\text {Metalaxyl }}+\Gamma_{\text {Atrazine }}(0.199) X_{\text {Atrazine }}+\Gamma_{\text {Malathion }}(0.216) \\
& X_{\text {Malathion }}-\Gamma_{\text {Prometryn }}(0.095) X_{\text {Prometryn }}-\Gamma_{\mathrm{Cu}}(1.22) \\
& X_{\mathrm{Cu}}+\Gamma_{\mathrm{Cd}}(2.91) X-\Gamma_{\mathrm{Pb}}(0.918) X_{\mathrm{Pb}}-\Gamma_{\mathrm{Zn}}(0.706) X_{\mathrm{Zn}}- \\
& \Gamma_{\mathrm{Ni}}(0.306) X_{\mathrm{Ni}}-\Gamma_{\text {Dimethoate* Metalaxyl }}(0.242) X_{\text {Dimethoate }} X_{\mathrm{Me}} \\
& \text { talaxyl }+\Gamma_{\text {Dimethoate*Atrazine }}(0.141) X_{\text {Dimethoate }} X_{\text {Atrazine }}+\Gamma_{\text {Dim }} \\
& \text { ethoate*Malathion }(0.072) X_{\text {Dimethoate }} X_{\text {Malathion }}+\Gamma_{\text {Dimethoate*Pro }} \\
& \text { metryn }(0.044) X_{\text {Dimethoate }} X_{\text {Prometryn }}+\Gamma_{\text {Dimethoate*Cu }}(0.063) \\
& X_{\text {Dimethoate }} X_{\mathrm{Cu}}-\Gamma_{\text {Dimethoate*Cd }}(0.170) X_{\text {Dimethoate }} X_{\mathrm{Cd}}+\Gamma_{\text {Dimethoate }} \\
& { }_{* \mathrm{~Pb}}(0.104) X_{\text {Dimethoate }} X_{\mathrm{Pb}}-\Gamma_{\text {Dimethoate* } \mathrm{Zn}}(0.043) X_{\text {Dimethoate }} X_{\mathrm{Zn}} \\
& +\Gamma_{\text {Dimethoate* } \mathrm{Ni}}(0.211) X_{\text {Dimethoate }} X_{\mathrm{Ni}}-\Gamma_{\text {Metalaxyl*Atrazine }}(0.068) \\
& X_{\text {Metalaxyl }} X_{\text {Atrazine }}-\Gamma_{\text {Metalaxyl*Malathion }}(0.238) X_{\text {Metalaxyl }} X_{\text {Malathi }} \\
& \text { on }+\Gamma_{\text {Metalaxyl*Prometryn }}(0.055) X_{\text {Metalaxyl }} X_{\text {Prometryn }}+\Gamma_{\text {Metalaxyl* }} \\
& { }_{\mathrm{Cu}}(0.121) X_{\text {Metalaxy1 }} X_{\mathrm{Cu}}-\Gamma_{\text {Metalaxyl*Cd}}(0.110) X_{\text {Metalaxy1 }} X_{\mathrm{Cd}}+\Gamma_{\mathrm{M}} \\
& \text { etalaxyl*Pb }(0.064) X_{\text {Metalaxyl }} X_{\mathrm{Pb}}-\Gamma_{\text {Metalaxyl*Zn }}(0.207) X_{\text {Metalaxyl }} X_{\mathrm{Z}} \\
& { }_{\mathrm{n}}+\Gamma_{\text {Metalaxyl* } \mathrm{Ni}}(0.087) X_{\text {Metalaxyl }} X_{\mathrm{Ni}}-\Gamma_{\text {Atrazine*Malathion }}(0.149) \\
& X_{\text {Atrazine }} X_{\text {Malathion }}+\Gamma_{\text {Atrazine* Prometryn }}(0.207) X_{\text {Atrazine }} X_{\text {Prometryn }}{ }^{-} \\
& \Gamma_{\text {Atrazine } * \mathrm{Cu}}(0.043) X_{\text {Atrazine }} X_{\mathrm{Cu}}+\Gamma_{\text {Atrazine }{ }^{* C d}}(0.255) X_{\text {Atrazine }} X_{\mathrm{Cd}^{-}}{ }^{-} \\
& \Gamma_{\text {Atrazine* }{ }^{\mathrm{Pb}}}(0.064) X_{\text {Atrazine }} X_{\mathrm{Pb}}-\Gamma_{\text {Atrazine*Zn }}(0.273) X_{\text {Atrazine }} X_{\mathrm{Zn}}{ }^{-} \\
& \Gamma_{\text {Atrazine*Ni}}(0.155) X_{\text {Atrazine }} X_{\mathrm{Ni}}-\Gamma_{\text {Malathion*Prometryn }}(0.159) X_{\mathrm{M}} \\
& \text { alathion } X_{\text {Prometryn }}+\Gamma_{\text {Malathion*Cu }}(0.168) X_{\text {Malathion }} X_{\mathrm{Cu}}+\Gamma_{\text {Malathion }}
\end{aligned}
$$

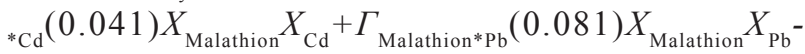

$$
\begin{aligned}
& \Gamma_{\text {Malathion*Zn }}(0.146) X_{\text {Malathion }} X_{\mathrm{Zn}}-\Gamma_{\text {Malathion*Ni }}(0.099) X_{\text {Malathio }} \\
& { }_{\mathrm{n}} X_{\mathrm{Ni}}+\Gamma_{\text {Prometryn*Cu}}(0.167) X_{\text {Prometryn }} X_{\mathrm{Cu}}+\Gamma_{\text {Prometryn*Cd }}(0.184) \\
& X_{\text {Prometryn }} X_{\mathrm{Cd}}+\Gamma_{\text {Prometryn*Pb }}(0.066) X_{\text {Prometryn }} X_{\mathrm{Pb}}+\Gamma_{\text {Prometryn }}
\end{aligned}
$$

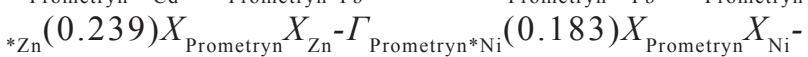

$$
\begin{aligned}
& \Gamma_{\mathrm{Cu}^{*} \mathrm{Cd}}(0.103) X_{\mathrm{Cu}} X_{\mathrm{Cd}}-\Gamma_{\mathrm{Cu} * \mathrm{~Pb}}(0.256) X_{\mathrm{Cu}} X_{\mathrm{Pb}}+\Gamma_{\mathrm{Cu}^{*} \mathrm{Zn}}(0.585) \\
& X_{\mathrm{Cu}} X_{\mathrm{Zn}}-\Gamma_{\mathrm{Cu}^{*} \mathrm{Ni}}(0.061) X_{\mathrm{Cu}} X_{\mathrm{Ni}}-\Gamma_{\mathrm{Cd} * \mathrm{~Pb}}(0.294) X_{\mathrm{Cd}} X_{\mathrm{Pb}}- \\
& \Gamma_{\mathrm{Cd} * \mathrm{Zn}}(0.244) X_{\mathrm{Cd}} X_{\mathrm{Zn}}-\Gamma_{\mathrm{Cd} * \mathrm{Ni}}(0.269) X_{\mathrm{Cd}} X_{\mathrm{Ni}}+\Gamma_{\mathrm{Pb} * \mathrm{Zn}}(0.313) \\
& X_{\mathrm{Pb}} X_{\mathrm{Zn}}+\Gamma_{\mathrm{Pb} * \mathrm{Zn}}(0.031) X_{\mathrm{pb}} X_{\mathrm{Ni}}-\Gamma_{\mathrm{Zn} * \mathrm{Ni}}(0.321) X_{\mathrm{Zn}} X_{\mathrm{Ni}}
\end{aligned}
$$

...where $Y_{(\mathrm{Cd})}$ is the adsorption capacity $(\mathrm{mg} / \mathrm{g})$ of $\mathrm{Cd}$ on the sediment; the independent variable, $X_{\dot{\mathrm{i}}}$, is the pollutant concentration level $(\mathrm{mol} / \mathrm{L}) ; X_{1} X_{\mathrm{j}}$ is the interaction of the two variables, $X_{\mathrm{i}}$ and $X_{\mathrm{j}}$ - which indicates that one of the independent variables on the dependent variable of the intensity depends on the concentration level of the other independent variables. The coefficient before each independent variable is expressed by $\Gamma_{\mathrm{i}}$. The results of the test of goodness of fitting to model (1) are shown in Table 6. The multiple correlation coefficients square $\left(\mathrm{R}^{2}\right)$ value in Table 6 is $87.91 \%$, which indicates that independent variables of model (1) can be used to explain the $\mathrm{Cd}$ adsorbed on the sediment, demonstrating that the fitting effect of model (1) is better. The forecast $\mathrm{R}^{2}$ obtained from repeat experiments was much lower than the correlation coefficients square $\left(\mathrm{R}^{2}\right)$, indicating that the model exhibits an overfitting phenomenon and some independent variables should not be introduced. As shown in Table 7 , the results of the $\mathrm{T}$ test for AT-MRAM was $>0.05$, confirming that the main effects and second-order interaction effects of the 10 types of factors that we studied in this paper had no significant impact on the adsorption of $\mathrm{Cd}$ onto the sediment. As a result, we established a new multiple linear regression model using the best subset regression modeling method to characterize the composite contamination condition of $\mathrm{Cd}$ and other pollutants adequately.

\section{Establishing the Best Subset Regression Model}

Best subsets regression is a method that can select an independent variable subset and identify the bestfitting regression models with user-specified predictors. The model was selected according to two standards, the maximum $\mathrm{R}^{2}$ and Mallows' $\mathrm{Cp}$, which estimates the mean square error to obtain as low a value as possible. In this study, the best subset regression model of Cd was established, and the output equation of each model was set to 2 to select the best subset combination.

As shown in Table 8, we should choose the eighth as the best subset regression model because its Mallows' Cp value is lowest and its adjusted $\mathrm{R}^{2}$ was more than $80 \%$. The best subset regression model was as follows:

$Y_{(\mathrm{Cd})}=9.57-\Gamma_{\text {Dimethoate }}(0.353) X_{\text {Dimethoate }}-\Gamma_{\mathrm{Cu}}(1.22)$ $X_{\mathrm{Cu}}+\Gamma_{\mathrm{Cd}}(2.91) X_{\mathrm{Cd}}-\Gamma_{\mathrm{Pb}}(0.918) X_{\mathrm{Pb}}-\Gamma_{\mathrm{Zn}}(0.706) X_{\mathrm{Zn}}-$ $\Gamma_{\mathrm{Ni}}(0.306) X_{\mathrm{Ni}}-\Gamma_{\mathrm{Cu} * \mathrm{~Pb}}(0.256) X_{\mathrm{Pb}} X_{\mathrm{Pb}}-\Gamma_{\mathrm{Cd} * \mathrm{~Pb}}(0.294) X_{\mathrm{Cd}} X_{\mathrm{Pb}}-$ $\Gamma_{\mathrm{Cd}^{*} \mathrm{Zn}}(0.244) X_{\mathrm{Cd}} X_{\mathrm{Zn}}-\Gamma_{\mathrm{Cd} * \mathrm{Ni}}(0.269) X_{\mathrm{Cd}} X_{\mathrm{Ni}}+\Gamma_{\mathrm{Pb} * \mathrm{Zn}}(0.313)$ $X_{\mathrm{Pb}} X_{\mathrm{Zn}}-\Gamma_{\text {Dimethoate*Metalaxyl }}(0.242) X_{\text {Dimethoate }} X_{\text {Metalaxyl }}{ }^{-}$ $\Gamma_{\mathrm{Zn} * \mathrm{Ni}}(0.321) X_{\mathrm{Zn}} X_{\mathrm{Ni}}+\Gamma_{\mathrm{Cu}^{*} \mathrm{Zn}}(0.585) X_{\mathrm{Cu}} X_{\mathrm{Zn}}$

\section{Conclusions}

The following conclusions can be drawn based on the results of our study:

1. In this study we used a resolution $\mathrm{V} 2^{10-3}$ fractional factorial experiment to filter out the significant main effects and the second-order interactive effects and characterize the combined degree of $\mathrm{Cd}$ pollution according to estimated effects. The adjusted $\mathrm{R}^{2}$ of the best subset regression model is more than $80 \%$; thus, the model can be used to predict the adsorption capacity of $\mathrm{Cd}$.

2. Compared with the resolution IV $2^{10-5}$ fractional factorial design method, the freedom of the resolution $\mathrm{V} 2^{10-3}$ fractional factorial design method increases from 21 to 45 . The total contribution to cadmium adsorption of the second-order interaction effect decreases from $61.48 \%$ to $12.92 \%$. This indicates that resolution IV overestimates the second-order interaction effect on cadmium adsorption on the sediments. 
3. This method can also be used to study the main effects and second-order interactions of other pollutants.

\section{Acknowledgements}

The authors are grateful for the financial support provided by the Ministry of Science and Technology of China ('973' Project No. 2004CB3418501).

\section{References}

1. KUMARASAMY M.V. Deoxygenation and reaeration coupled hybrid mixing cells based pollutant transport model to assess water quality status of a river. Int. J. Environ. Res. 9 (1), 341, 2015.

2. UDEIGWE T.K., TEBOH J.M., EZE P.N. Implications of $\mathrm{Pb}$ crop production practices on environmental quality and human health. J. Environ. Manage. 151, 267, 2015.

3. D'AMATO G., CECCHI L., D'AMATO M. Urban air pollution and climate change as environmental risk factors of respiratory allergy: an update. J. Invest. Allerg. Clin. 20(2), 95, 2010.

4. CONCAS A., ARDAU C., CRISTINI A. Mobility of heavy metals from tailings to stream waters in a mining activity contaminated site. Chemosphere. 63 (2), 244, 2006.

5. GOVIL P.K., SORLIE J.E., MURTHY N.N. Soil contamination of heavy metals in the Katedan industrial development area, Hyderabad, India. Environ. Monit. Assess. 140 (1-3), 313, 2008.

6. CLEMENS S. Toxic metal accumulation, responses to exposure and mechanisms of tolerance in plants. Biochimie. 8, (11), 1707, 2006.

7. SINGER R.S., COX L.A., DICKSON J.S. Modeling the relationship between food animal health and human foodborne illness. Prev. Vet. Med. 79 (2-4), 186, 2007.

8. SUN Z. G., WANG L. H., ZHOU Q. Effects and mechanisms of the combined pollution of lanthanum and acid rain on the root phenotype of soybean seedlings. Chemosphere. 93 (2), 344, 2013.

9. DONG D.M., NELSON Y.M., LION L.W. Adsorption of $\mathrm{Pb}$ and $\mathrm{Cd}$ onto metal oxides and organic material in natural surface coatings as determined by selective extractions: new evidence for the importance of $\mathrm{Mn}$ and Fe oxides. Water. Res. 34 (2), 427, 2000.

10. LI Y., WANG X.L., WANG Y. Comparison of $\mathrm{Pb}$ and $\mathrm{Cd}$ adsorption to the surface coatings and surficial sediments collected in Xianghai Wetland. J. Environ. Sci. 17 (1), 126, 2005.

11. VELAZQUEZ M.A., LUIS P.J., ORTEGA M. Study of the distribution of boron in water sources of the Duero river basin, Mexico, using multivariate statistical analysis. Rev. Int. Contam. Ambie. 27 (2), 19, 2011

12. FOSSO-KANKEU E., MULABA-BAFUBIANDI A.F. MAMBA B.B. Prediction of metal-adsorption behaviour in the remediation of water contamination using indigenous microorganisms. J. Environ. Manage. 92 (10), 2786, 2011.

13. NOVAKOVA T., MATYS G.T., BABEK O. Distinguishing regional and local sources of pollution by trace metals and magnetic particles in fluvial sediments of the Morava River, Czech Republic. J. Soil. Sediment. 13 (2), 460, 2013.

14. LY S.S., GAO Q., WANG X.L. Using multiple regression adsorption model to estimate $\mathrm{Zn}$ and $\mathrm{Cu}$ adsorptions onto $\mathrm{Fe}$ oxides, Mn oxides, organic materials and their blends in surficial sediments. Fresen. Environ. Bull. 19 (8), 1466, 2010.

15. RUIZ F., GONZALEZ-REGALADO M.L., MUNOZ J.M. Distribution of heavy metals and pollution pathways in a shallow marine shelf: assessment for a future management. Int. J. Environ. SCI. Te. 11 (5), 1249, 2014.

16. GUO Z.Y., DONG D.M., HUA X.Y., ZHANG L.W., ZHU S.J., LAN X.H., LIANG D.P. Cr and As decrease lindane sorption on river solids. Environ. Chem. Lett. 13 (1), 111, 2015.

17. LI Y., CHENG B.C., LI S.S., WANG M. Investigation on the combined pollution between malathion with a variety of pollutants based on the fractional factorial design (Resolution IV). Pol. J. Environ. Stud. 24 (5), 2015. (In press)

18. CLAUDIOA S.R., GOLLUCKEA A.P.B., YAMAMURA H., MORAISB D.R., BATAGLIONB G.A., EBERLINB M.N., PERESA R.C., RIBRIRO D.A. Purple carrot extract protects against cadmium intoxication in multiple organs of rats: Genotoxicity, oxidative stress and tissue morphology analyses. J. Trace. Elem. Med. Bio. 33 37, 2016 [In Press].

19. JARUP L., AKESSON A. Current status of cadmium as an environment health problem. Toxicol. Appl. Pharmacol. 238 (3), 201, 2009.

20. WANG D.X., COMERLY M.D. Evaluating the power of Minitab's data subsetting lack of fit test in multiple linear regressions. J. Appl. Stat. 35 (1), 115, 2008.

21. LI Y., MA D.C., GAO Z.T., CHENG B.C., ZHAO W.J., WANG M. Characristic of competitive adsorption between cadmium and a variety of pollutants based on fractional factorial design (Resolution IV). Sci. Technol. Eng. 14 (20), 1671, 2014 [In Chinese]. 